\section{The Effect of an Acute}

\section{Sedentary Behaviour}

\section{Reducing Intervention}

on Subjective Well-Being

among University Students:

A Pilot Randomized Trial

WUYOU SUI (D)

HARRY PRAPAVESSIS (1)

*Author affiliations can be found in the back matter of this article

\section{] $\mathrm{u}$ [ubiquity press}

\begin{abstract}
Background: The effect of sedentary behaviour (SB) on subjective well-being (SWB), particularly through a SB-reducing intervention largely remains unknown. This pilot trial examined whether an acute intervention designed to reduce SB would enhance SWB in a sample of university students.
\end{abstract}

Methods: A three-week (i.e., baseline, intervention, follow-up) randomized controlled pilot trial was conducted. Thirty-two sedentary university students were randomized to an acute behavioural counseling intervention $(n=17)$ or control group $(n=15)$. Behavioural counseling grounded in the health action process approach aimed at reducing daily SB for 1 week. Device-measured outcomes (i.e., steps, standing, sitting, sit-to-stand transitions), self-reported SBs (i.e., self-compared, domain-specific), and SWB measures (i.e., affect, life satisfaction, subjective vitality, overall SWB) were assessed weekly.

Results: Repeated-measures ANOVAs revealed non-significant medium-to-large effects for self-reported SBs (i.e., $0.116 \leq \eta_{p}{ }^{2} \leq 0.253$ ), device-measured standing time (i.e., $\eta_{p}{ }^{2}=0.161$ ), and life satisfaction and overall SWB (i.e., $0.141 \leq \eta_{p}{ }^{2} \leq 0.178$ ) favouring the treatment group over the control group.

Conclusions: Overall, this acute intervention was ineffective in reducing SB among university students. Comparatived to previous acute SB-inducing interventions, results suggest that SB-reducing interventions may require more robust treatment application than the current pilot study. Strategies such as prompts/cues, repeated intervention delivery, and longer intervention periods are recommended. Strategies that promote larger non-convenient sampling (e.g., longer recruitment periods) also are recommended. Taken together, these strategies will increase treatment effects and statistical power of subsequent intervention trials.
CORRESPONDING AUTHOR:

\section{Wuyou Sui}

Western University, CA

wsui@uvic.ca

\section{KEYWORDS:}

sedentary behaviour; subjective well-being; university students; pilot trial; health action process approach

TO CITE THIS ARTICLE: Sui, W \& Prapavessis, $\mathrm{H}$. (2021). The Effect of an Acute Sedentary Behaviour Reducing Intervention on Subjective Well-Being among University Students: A Pilot Randomized Trial. Health Psychology Bulletin, 5(1), pp. 46-60. DOI: https://doi.org/10.5334/hpb.29 


\section{INTRODUCTION}

Sedentary behavior (SB) describes any behavior that is (i) waking, (ii) has an energy expenditure of $\leq 1.5$ metabolic equivalents, and (iii) is performed in a sitting, lying, or reclining posture (Tremblay et al., 2017), and encompass the vast majority of behaviors performed in nearly every domain of daily life. Given that most individuals spend the bulk of their waking time engaged in SBs (Colley et al., 2011), particular focus has been devoted to studies investigating the health consequences of excessive SB, such as heart disease, type 2 diabetes, hypertension, some cancers, and all-cause mortality (De Rezende et al., 2014). Notably, relatively fewer studies have examined SB and health through a salutogenic lens, whereby health encompasses "positive health conceptions, such as quality of life, flourishing, and well-being" (Mittelmark \& Bauer, 2017). One salutogenic outcome of particular interest is subjective well-being (SWB).

Subjective well-being, as defined by Diener et al., is "a broad category of phenomena that includes people's emotional responses, domain satisfactions, and global judgements of life satisfaction", which is assessed through outcomes of affect (positive and negative) and life satisfaction (1999). This operationalization of SWB by Diener et al. (1999), is considered hedonic well-being, whereby optimal SWB is attained through maximizing happiness (Ryan \& Deci, 2001). An interfacing conceptualization of SWB is eudaimonic well-being, whereby optimal SWB is attained through fulfillment of purpose and self-realization (Huta, 2015).

Previous works examining the relationship between SWB and SB demonstrate mixed results. (Hogan et al., 2015; Wrosch \& Sabiston, 2013). Results from experimental studies suggest that greater SB results in lower positive affect (Duvivier et al., 2017), greater negative affect (Edwards \& Loprinzi, 2016; Endrighi et al., 2016), and lower life satisfaction (Edwards \& Loprinzi, 2017). However, all of these studies experimentally increased SB, in that participants in the treatment group were encouraged to sit more and move as little as possible for a period of four days (Duvivier et al., 2017) to two weeks (Endrighi et al., 2016). Additionally, experimental works examining SB and SWB have not consistently measured SB as is currently defined (Tremblay et al., 2017), instead using accelerometers (Endrighi et al., 2016) or step counters (Edwards \& Loprinzi, 2016, 2017) as a proxy for SB. Issues with previous works are further confounded when considering their lack of measurement of domain-specific SB. Domain-specific SB, or the SB accumulated in different contexts of sitting (e.g., sitting during meals, occupation, screen time, etc.), demonstrate stronger, and often opposite, associations (i.e., rs) with outcomes of SWB than total SB (O'Neill \& Dogra, 2016). Thus, measurement of domain-specific sitting may reveal unique relationships with outcomes of SWB, independent of total sitting.
Overall, previous work has been limited by either cross-sectional design, solely increasing SB, invalid measurement of SB, and/or lack of delineation between total and domain-specific sitting behaviors. Hence, there is the need for a study that experimentally decreases SB and assesses device-measured and domain-specific SB to evaluate the effect of SB on outcomes of SWB. Notably, the use of explicit behavioral theory is absent among SBinducing studies. One such theory is the Health Action Process Approach (HAPA; Schwarzer, 2008). The HAPA delineates individuals' behaviour change status into three stages: preintenders, intenders, and actors, with specific constructs predicting and mediating progression through the stages. Most notably, the HAPA postulates that action and coping planning mediate the 'gap' between intention and behaviour (i.e., action). Application of the HAPA model in health behaviour change has been used to predict the adoption and maintenance of physical activity (Aliabad et al., 2014; Lippke et al., 2005) and dietary behaviours (Y. P. Duan et al., 2017). With respect to SB specifically, the HAPA model for behavior change has shown suitability and has shown success in increasing break frequency among a sample of university students (Sui \& Prapavessis, 2018) and increasing non-SBs among office workers (Rollo \& Prapavessis, 2020).

Therefore, a randomized pilot trial was conducted to experimentally explore whether an intervention designed to decrease SB will lead to improvements in SWB. The main objective of this trial was to determine the preliminary effectiveness of an acute HAPA-based behavioral intervention to decrease SB and increase SWB among sedentary university students, in order to inform a future randomized controlled trial. A secondary objective of this trial was to explore whether any changes in SB outcomes would be related to changes in outcomes of SWB.

\section{METHODS}

Trial reporting is guided by the CONSORT 2010 Statement for reporting randomized control trials (Moher et al., 2012). A completed CONSORT checklist can be found in Supplementary Material 1.

\section{TRIAL DESIGN/ETHICS}

A three-week (baseline, intervention, post-intervention), single-blinded, parallel-group (equal allocation ratio [1:1]), randomized-controlled pilot trial was conducted. No changes to trial protocol, eligibility criteria, or planned statistical analyses were made after trial commencement. Ethics approval for the study was obtained from the host institution's REB (Project ID: 112399).

\section{PARTICIPANTS/RECRUITMENT}

A convenience sample of university students from the host institution were recruited to participate in this study 
through posters distributed around university campus, verbal advertisement during lectures, and through online university groups on Facebook. Eligible participants (a) were full-time university students attending the host institution, (b) aged 18 years of age or older, (c) who were able to read and write in English, (d) had access to a computer/smartphone with internet, and (e) recorded $\geq 7$ hours of sedentary time per day over the baseline week, via ActivPAL4 inclinometer. The cut-off time of seven hours of sedentary time per day was based off metaregression findings regarding self-reported SB by Po-Wen and colleagues (Ku et al., 2018). Exclusion criteria were (a) part-time enrollment or currently on a leave of absence from full-time studies at university, (b) individuals selfreporting a mental illness, and (c) individuals currently reporting a physical disability that would prevent them from walking.

\section{SETTING/DATA COLLECTION PERIOD}

The study took place at a mid-sized ( 30,000 students) post-secondary institution in Ontario, Canada during the 2019-2020 academic year.

\section{INTERVENTION TREATMENT}

Participants randomly allocated to the intervention group were told they would receive a single theorydriven behavioral counseling session, with the goal of codeveloping strategies aimed at (1) decreasing their weekly SB by 1-2 hours, as well as (2) increasing their daily step count to $\geq 10,000$ steps/day, over the next week.

Participants were initially prompted to think about strategies to reduce their SB and increase steps/day, followed by the researcher stating: "strategies that we come up with should be specific, so that you're not thinking about when, where, or how you're going to do them, or who you're going to do them with. Strategies also should be realistic for you, because if you don't find them realistic, you're probably not going to do them". Participants were then asked if they had any strategies that they could immediately think of. Prompts for behavioral strategies were used if the participant could not think of any strategy, and generally revolved around reducing SB in a particular domain of SB (e.g., transportation, occupation, etc.).

Creation of behavioral strategies was guided by the FITT principle (Burnet et al., 2020), adapted to a SB context; where $F$ represented the frequency per week that a strategy would be enacted (e.g., 3-4 times per week [Monday, Wednesday, Friday], every hour of sitting); I represented the intensity/length of time that a strategy be performed for (e.g., 20 minutes, 5-minute break from sitting, 3000 steps); the first $T$ represented the time of day that the behavior would be be performed (e.g., 6PM, mornings [8-11], during studying); and the second T represented the type (i.e., modality) of behavior performed (e.g., standing, walking, weightlifting).

Upon creation of a strategy and its FITT specifics, an accompanying coping strategy (or strategies) was prompted via the researcher stating: "With any new strategy that we may try and implement, there are inevitably barriers that would prevent us from enacting the strategy. Can you think of any barriers or reasons that you might not perform this strategy? What could you do or plan for so that you could still perform the strategy, despite these barriers?". Coping strategies often focused on practical steps, like setting an alarm or reminding a friend to work out together. Proceeding creation of a complete strategy, participants were asked if they thought the strategy was specific and realistic enough as a fidelity check. Upon agreement, participants were prompted to think about any other strategies that could help them achieve the SB and step count goals for the next week. Strategies also often included using the native step counter app on their smartphone (e.g., Health on iOS, Google Fit on Google devices) to selfmonitor their daily step count. At the end of the session, participants were encouraged to try each strategy at least once, and to try to adjust strategies if unperceived barriers arise. Additionally, participants were advised to place the completed counseling form somewhere where they would regularly see it. On average, participants codeveloped 3-4 strategies. Behavioral counseling session typically took 30-45 minutes and were delivered face-toface by the researcher in the researcher's lab.

Behavioral counseling was guided by the HAPA (Schwarzer, 2008) with associated intervention behavior change techniques labelled as (1.1) Goal Setting (behavior); (1.2) Problem Solving; (1.4) Action Planning; (1.5) Review behavior goal(s); (1.9) Commitment; (2.3) Self-monitoring of behaviors; and (7.1) Prompts and cues, according to the Michie et al., (2013) behavior change taxonomy. Prior application of the HAPA model and the aforementioned behavior change techniques to manipulate SB in this population has also been utilized in previous work (Sui \& Prapavessis, 2018).

\section{Control}

Participants randomly allocated to the control group received no specific instructions to modify their behavior. If prompted for further instruction, the researcher would encourage the participant to continue their normal behavior.

\section{PRIMARY OUTCOMES}

\section{Device-measured SB/PA}

The ActivPAL4 inclinometer (PAL Technologies, 2020) was used to track the device-measured PA and SB of participants. A 7-day 24-hour continuous wear protocol was used. The ActivPAL4 was attached to the center of the right thigh, halfway between the superior iliac spine 
of the hip and the patella (Edwardson et al., 2017). Participants did not take off the device during bathing or water-based activities. Given the 7-day 24-hour wear protocol, a minimum of 6 valid days (i.e., 144 hours) of wear time was deemed necessary to be included in analysis to be representative of the week. Collected data were visualized through graphs and scanned for abnormalities (e.g., excessively large volumes or high intensities of activity). Outcomes derived from the ActivPAL4 device included: average daily steps (steps per day); average daily standing time (minutes per day); average daily stepping time (minutes per day); average daily sitting time (minutes per day); and average daily sit-to-stand transitions (transitions per day).

\section{Self-reported SB}

Past 7 days self-compared SB (i.e., sitting, break frequency, break duration) was assessed through three items on a 5-point Likert scale ("In the last 7 days..."), with a score of 1 indicating Much less than normal, 3 indicating About the same, and 5 indicating Much more than normal.

Past 7 days domain-specific SB was assessed using the modified SIT-Q 7d questionnaire (Sui \& Prapavessis, 2016). The original SIT-Q 7d instrument (Wijndaele et al., 2014) is a self-reported questionnaire that measures weekday (WY) and weekend (WD) time spent sitting in various activities in a number of domains of activity over the past 7 days (i.e., sleep and naps, meals, transportation, screen time, occupation(s), and other activities). A sample questionnaire can be found in Supplementary Material 2. Domain-specific SB question options were recoded to represent the upper limit (i.e., more sedentary) of that option, in order to signify the most conservative estimate of SB (e.g., '15-30 min recoded to 0.5 hours).

Past 7 days total weekday SB was assessed through a single question on the International Physical Activity Questionnaire - Short Form 7 days (IPAQ-S7S; Craig et al., 2003). Participants were asked to specify both an hour(s) per day and minute(s) per day estimate. A total weekly sitting estimate was calculated by multiplying responses by seven.

\section{Outcomes of SWB}

Past 7 days positive and negative affect was assessed through the Positive and Negative Affect Schedule (PANAS; Watson et al., 1988). Separate scores (range: 10-50) for positive and negative affect are attained by summing items assessing positive and negative affect, respectively.

Life satisfaction was assessed through the Satisfaction with Life Scale (SWLS: Diener et al., 1985). A total score (range: 7-35) is attained by summing the responses, with higher scores representing higher life satisfaction.

Eudaimonic well-being was assessed through the Subjective Vitality Scale (SVS; Ryan \& Frederick, 1997). A shorter, more validated, six question version of the SVS developed by Bostic, Rubio, \& Hood was used in the present work (Bostic et al., 2000).
Overall SWB was assessed through the WarwickEdinburgh Mental Well-Being Scale (WEMWBS; Tennant et al., 2007). A total score (range: 14-70) is attained by summing the responses, with higher scores representing greater subjective well-being.

\section{OTHER OUTCOMES}

Demographics were assessed through a single item (e.g., "What is your..."); age, preferred gender, current program of study, current year of study, degree pursuing, and ethnicity were collected. Past 7 days physical activity was assessed using the IPAQ-S7S (Craig et al., 2003). A total weekly activity score for each physical activity intensity was attained through the product of how many days that exercise was performed, and average hours spent in performing said intensity of exercise.

\section{PROCEDURE}

Interested participants met with the researcher, who presented the detailed letter of information and consent and collected consent. Consenting participants then completed the demographics questionnaire and then were fitted with the ActivPAL4 inclinometer. Participants then scheduled their second visit for seven days after the initial meeting. At the beginning of the second visit, the ActivPAL4 was removed and SB levels for inclusion were verified. Upon confirmation of eligibility, participants were assigned to either the treatment or control group. Participants then completed the online questionnaires, beginning with the self-compared sitting questions, and followed by the modified SIT-Q 7d questionnaire, IPAQS7S, PANAS, the SWLS, the SVS, and the WEMBWS. After the questionnaires, participants in the treatment group received the single session of behavioral counseling, while those in the control group received no behavioral instructions. Participants in both groups were then refitted with a new ActivPAL4 device and scheduled their third visit for a week later. During the third visit, the ActivPAL4 device was removed and both groups completed the questionnaires for the second time. Afterwards, a new ActivPAL4 device was refit and participants were given no specific behavioral instructions (treatment group participants were told to "do whatever you want" if the researcher was asked for instructions), and the fourth visit was scheduled for a week in the future. During the fourth visit, ActivPAL4 devices were removed from participants, and all participants completed the questionnaires for the third time. A researcher then debriefed all participants. Participants received a $\$ 30$ gift card for their participation.

\section{REQUIRED SAMPLE SIZE}

Due to the pilot nature of the present trial, no formal sample size calculation was used. Rather, the sample size of previous acute SB intervention studies was used as a guideline for sample size (Duvivier et al., 2017; Edwards \& 
Loprinzi, 2017). Hence, a sample size of 30 was deemed appropriate for an initial pilot.

\section{RANDOMIZATION}

Block randomization was used to allocate participants to either intervention or control groups in a 1:1 ratio with a fixed block size of 36 participants (i.e., 18 intervention and 18 control, accounting for a 20\% attrition rate based on a previous intervention in this population; (Sui \& Prapavessis, 2018). The random number sequence was generated through the list randomizer on RANDOM. org (RANDOM.org, 2020). Allocation was not concealed to the researchers. The same researcher generated the random allocation sequence, enrolled participants, and assigned participants to groups.

\section{BLINDING}

Participants were blinded to the intervention content and assignment. The study was advertised as a "behavior change study" in recruitment materials, in the letter of information/consent, and in participant correspondence.

\section{STATISTICAL ANALYSIS}

Independent-samples $t$-tests were used to compare baseline demographic characteristics and primary outcome variables between groups to determine adequate group randomization. A series of 2 (treatment vs. control) $\times 3$ (time: visit 2 , visit 3 , visit 4 ) repeated measures ANOVAs were used to identify any time by group interaction effects and was accompanied by partial eta squared $\left(\eta_{p}{ }^{2}\right)$ and observed power values. Bivariate correlations (absolute and residual change) were computed for intervention-baseline differences (i.e., visit 3 - visit 2) between SB outcomes and outcomes of SWB to affirm relationships between these outcomes. For certain device-based SB measures (i.e., average daily standing time, average daily stepping time, average daily sitting time, and average daily sit-to-stand transitions), interaction effects and correlations were calculated using the percentage of the waking day these behaviours were performed (e.g., percent of the waking day spent sitting). Analysis was computed using IBM SPSS software (version 23). Statistical significance was set at .05. Partial eta squared, confidence intervals, and statistical power accompanied all $p$ values. In line with Bakeman (2005), partial eta squared values of $0.02,0.13$, and 0.26 represented small, medium, and large effect size statistics for repeated measure designs.

\section{DATA IMPUTATION}

A Winsorization technique was applied to the data; data points over the $95^{\text {th }}$ percentile and underneath the $5^{\text {th }}$ percentile in each group were replaced with the value of the $95^{\text {th }}$ percentile and $5^{\text {th }}$ percentile, respectively. Winsorization has demonstrated validity as a method for dealing with outliers (B. Duan, 1998). A total of 63 data points (1.00\% of possible data points) were imputed this way.

\section{RESULTS \\ SAMPLE CHARACTERISTICS}

\section{Missing Data}

Two participants who were assessed for eligibility were excluded. One participant was excluded for not meeting the SB inclusion threshold (i.e., reported $<7$ hours/day of $\mathrm{SB}$ ), while the other participant chose not to participate citing the time commitment. Flow of participants and dropouts is shown in Figure 1.

\section{Recruitment}

Participants were recruited during the Fall 2019 and Winter 2020 academic terms at the host institution (i.e., September 2019 to February 2020). The study was stopped after planned sample size of completed participants was reached.

\section{Group Equivalency}

Sample characteristics are presented in Table 1. Significant group differences at baseline were observed for average daily steps, average daily stepping time, WY sleep time, and WY napping, whereby the treatment group reported significantly higher in all outcomes (ps > 0.05). For these variables, baseline scores were input as covariates to account for differences at baseline. No other significant differences between groups at baseline were found.

\section{Descriptive Data}

Descriptive data for device-measured SB outcomes are presented in Table 2. Descriptive data for self-compared SB, average weekday sitting are presented in Table 3. Descriptive data for domain-specific SB and outcomes of SWB are presented in Supplementary material 3 and Table 4, respectively.

\section{INTERACTION EFFECTS AND MAIN EFFECTS}

Interaction effects and main effects for device-measured SB outcomes are presented in Table 5. Overall, no devicemeasured SB outcome demonstrated a significant interaction effect; however, a significant main effect for average daily standing time was observed ( $p=0.004$ ), indicating that both groups increased their percentage of stepping time during their day from baseline to intervention and then returned to baseline levels post-intervention.

Interaction effects and main effects for self-compared SB outcomes are presented in Table 5. Overall, no selfcompared SB outcome demonstrated a significant interaction effect. Additionally, a significant main effect for self-compared break frequency was observed, 


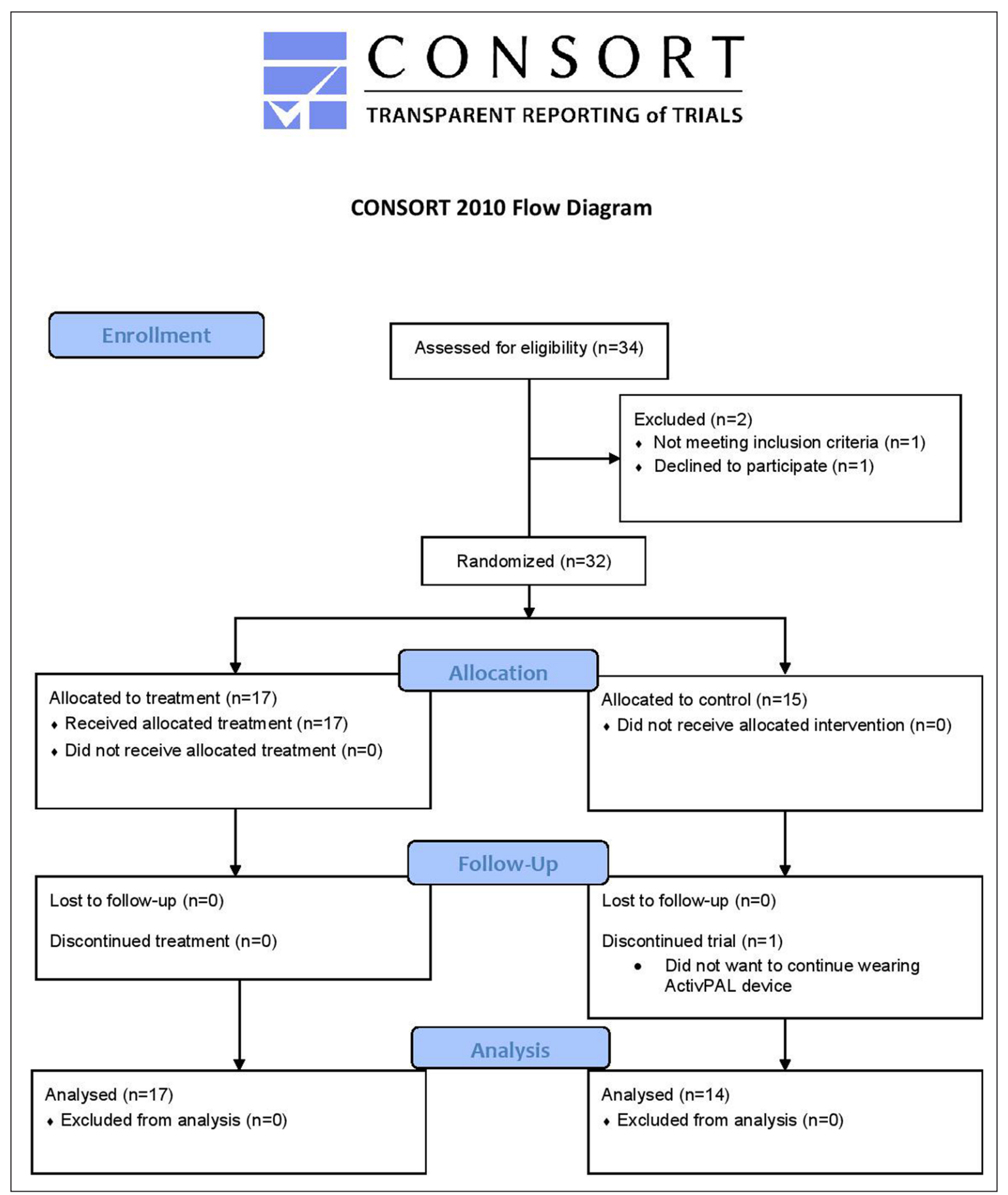

Figure 1 Flow of participants through the study.

indicating both groups perceived an increase in their break frequency from baseline to intervention and then a drop back to baseline post-intervention.

Interaction effects and main effects for self-reported domain-specific SB are presented in Supplementary material 4. Overall, only WY sleep $[F(1,28)=5.135$, $p=0.031]$ and average number of breaks from other activities demonstrated a significant interaction effect $[F(2$, $28)=4.737, p=0.017]$. This corresponded to an increase in sleep time for the treatment group and decrease in sleep time for the control group from intervention to postintervention and both groups decreasing their number of breaks from baseline to intervention, but only the treatment group increasing their other activities break frequency back to baseline levels post-intervention. Significant main effects ( $p \leq 0.05$ ) were also observed for WD napping, WY lunch, total weekly transportation, student break duration, WY and WD TV, WD computer/smartphone use, screen time break frequency and duration, and other activities break frequency. For WY lunch, total weekly transportation, WYI WD TV, and WD computer use, this was associated with both groups decreasing these SBs from baseline to postintervention. For break frequency and duration measures, this was associated with a decrease in break frequency and break duration for both groups from baseline to postintervention. 


\begin{tabular}{|c|c|}
\hline & $M(S D)$ \\
\hline Age & $19.45(2.68)$ \\
\hline Gender & $n(\%)$ \\
\hline Men & $12(38.7)$ \\
\hline Women & $19(61.3)$ \\
\hline Year of Study & $n(\%)$ \\
\hline First Year & $13(41.9)$ \\
\hline Second Year & $11(35.5)$ \\
\hline Third Year & $2(6.5)$ \\
\hline Fourth Year or Higher & $4(12.9)$ \\
\hline Missing & $1(3.2)$ \\
\hline Current Degree Pursuing & $n(\%)$ \\
\hline Undergraduate & $30(96.8)$ \\
\hline Doctorate or Professional degree & $1(3.2)$ \\
\hline Current Program of Study & $n(\%)$ \\
\hline Engineering & $2(6.5)$ \\
\hline Health Sciences & $19(61.3)$ \\
\hline Information and Media Studies & $2(6.5)$ \\
\hline Science & $5(16.1)$ \\
\hline Social Sciences & $2(6.5)$ \\
\hline Business and Finance & $1(3.2)$ \\
\hline Ethnicity & $n(\%)$ \\
\hline European/Caucasian & $7(22.6)$ \\
\hline Canadian & $2(6.5)$ \\
\hline Hispanic & $1(3.2)$ \\
\hline Asian & $14(45.2)$ \\
\hline Black & $3(9.7)$ \\
\hline
\end{tabular}

Table 1 Demographic Characteristics $(n=31)$.

Interaction and main effects for SWB outcomes are presented in Table 5. Overall, no outcome of SWB demonstrated a significant interaction effect.

\section{RESIDUAL CHANGE AND ABSOLUTE DIFFERENCES CORRELATIONS}

Correlations between change in device-measured SB outcomes and change in outcomes of SWB are presented in Table 6. Change in average daily standing time was significantly correlated with absolute change in overall SWB ( $p \leq 0.05)$. Correlations between change in self-compared and average weekday SB outcomes and change in outcomes of SWB are presented in Table 7. Residual change in self-compared weekly break frequency was significantly positively correlated with life satisfaction and overall SWB ( $p s \leq 0.05$ ). Change in self-compared weekly break duration was significantly positively correlated with residual change in life satisfaction, as well as absolute change in positive affect, life satisfaction, subjective vitality, and overall SWB ( $p$ s $\leq 0.05$ ). Significant negative correlations were observed between change in average weekday sitting and both residual change and absolute change in life satisfaction ( $p s \leq 0.05$ ). Correlations between change in domainspecific SB and change in outcomes of SWB are presented in Supplementary Material 5. Notable significant domainspecific correlations included sleep, screen time, other activities, and breaks from sitting ( $p s \leq 0.05$ ).

\section{ADVERSE EVENTS}

Given the minimal risk associated with the intervention and subsequent behaviors, data regarding adverse events/ harms were not formally collected. However, anecdotally, no participants noted any adverse events or harms.

\section{DISCUSSION \\ INTERVENTION EFFECTIVENESS - SEDENTARY BEHAVIOR}

Overall, the intervention was unable to significantly decrease device-measured SB, relative to the control group. Low observed power statistics provide evidence that the trial was underpowered to observe changes in these device-measured SB outcomes. However, a medium-sized effect size for average daily standing time was observed $\left(\eta_{p}{ }^{2}=0.161\right)$. An informal sample size calculation suggests that a sample size of 60 participants would be necessary to see significant changes in device-measured average standing time. In contrast to device-measured SB, the effect of the intervention on self-reported SBs was more encouraging. For selfcompared SB, specifically, consistent medium-sized interaction effects that favoured treatment were observed (i.e., $0.140 \leq \eta_{p}^{2} \leq 0.183$ ). Similarly, selfreported average weekday sitting and several domainspecific SBs demonstrated medium-to-large effect sizes (i.e., $0.116 \leq \eta_{p}{ }^{2} \leq 0.253$ ) in favor of the treatment group. Average number of breaks from sitting during other activities, in particular, showed a significant interaction effect in favor of the treatment group, indicating that the treatment group took more breaks from other activities (e.g., hobbies, reading) than the comparison group. These findings highlight the disparity between how sedentary university students perceive they are and their actual device-measured behaviour (Castro et al., 2020), as well as potential self-report bias among the intervention group.

The (lack of) effectiveness of the current SB-reducing intervention presents a stark contrast to the effectiveness of previous SB-inducing studies. Compared to the effectiveness of previous SB-inducing studies like Endrighi and colleagues (i.e., $31.48 \mathrm{~min} /$ day increase in sitting time, 95\% CI: [-57.64, -5.32]; 2016), or Duvivier and colleagues 


\begin{tabular}{|c|c|c|c|c|c|c|}
\hline & \multicolumn{3}{|c|}{ TREATMENT $(n=17)$} & \multicolumn{3}{|c|}{ CONTROL $(n=14)$} \\
\hline TIME & MEAN & $S D$ & $95 \% C I$ & MEAN & $S D$ & $95 \% C I$ \\
\hline \multicolumn{7}{|c|}{ Average Daily Steps (steps/day) } \\
\hline Baseline & 9252.54 & 1925.78 & {$[8262.40,10242.78]$} & 7328.67 & 2325.12 & {$[5986.18,8671.15]$} \\
\hline Intervention & 11028.20 & 2836.38 & {$[9569.87,12486.53]$} & 8485.20 & 3020.25 & {$[6741.36,10229.05]$} \\
\hline Follow-up & 9689.50 & 2714.05 & {$[8294.06,11084.93]$} & 7478.33 & 2969.84 & {$[5763.59,9193.06]$} \\
\hline \multicolumn{7}{|c|}{ Average Daily Standing Time (minutes/day) } \\
\hline Baseline & 188.74 & 47.03 & {$[164.56,212.92]$} & 189.06 & 62.63 & {$[152.90,225.22]$} \\
\hline Intervention & 192.32 & 41.25 & {$[171.12,213.53]$} & 190.55 & 60.23 & {$[155.77,225.32]$} \\
\hline Follow-up & 189.81 & 53.64 & {$[162.23,217.39]$} & 216.70 & 72.45 & {$[174.87,258.53]$} \\
\hline \multicolumn{7}{|c|}{ Average Daily Stepping Time (minutes/day) } \\
\hline Baseline & 108.75 & 24.42 & {$[96.20,121.31]$} & 85.53 & 22.48 & {$[72.55,98.51]$} \\
\hline Intervention & 125.31 & 33.79 & {$[107.93,142.68]$} & 96.50 & 30.70 & {$[78.78,114.23]$} \\
\hline Follow-up & 112.54 & 31.35 & {$[96.42,128.66]$} & 89.00 & 31.91 & {$[70.58,107.42]$} \\
\hline \multicolumn{7}{|c|}{ Average Daily Sitting Time (minutes/day) } \\
\hline Baseline & 660.11 & 80.26 & {$[618.84,701.37]$} & 731.20 & 92.96 & {$[677.52,784.87]$} \\
\hline Intervention & 655.57 & 83.80 & {$[612.48,698.66]$} & 722.96 & 105.47 & {$[662.06,783.86]$} \\
\hline Follow-up & 651.36 & 81.78 & {$[609.31,693.41]$} & 707.85 & 71.69 & {$[666.46,749.24]$} \\
\hline \multicolumn{7}{|c|}{ Average Daily Sit-to-Stand Transitions (number/day) } \\
\hline Baseline & 50.31 & 14.20 & {$[43.01,57.61]$} & 44.79 & 13.87 & {$[36.78,52.80]$} \\
\hline Intervention & 48.84 & 11.99 & {$[42.67,55.00]$} & 43.22 & 12.56 & {$[35.97,50.48]$} \\
\hline Follow-up & 49.43 & 11.99 & {$[43.26,55.59]$} & 45.04 & 13.80 & {$[37.07,53.01]$} \\
\hline
\end{tabular}

Table 2 Means, standard deviations, and 95\% confidence intervals for device-measured sedentary behaviors throughout the study. Note: Bold text indicates significant differences between groups at baseline $(p<0.05)$.

(e.g., +5.9 hours/day increase in sitting time, 95\% CI: [5.75, 6.05]; 2017), the present work observed a modest 6.21 $\mathrm{min} /$ day (95\% CI: $[-23.19,35.61])$ decrease in sitting time for the treatment group over a 1-week intervention. Despite the contrast in effectiveness between the present work and prior SB-inducing work, comparisons between the two are misleading and inappropriate. Firstly, the present study used inclinometry to measure SB, compared to the use of accelerometers in the previously mentioned work to measure 'sedentary time' (i.e., physical inactivity), which cannot distinguish between sitting and standing nor sensitive enough to capture postural changes (Koster et al., 2016). Secondly, inducing SB likely warrants a different approach to intervention than reducing $S B$ given the habitual nature of SB, as behavior modification through rewarding/reinforcing the habit behavior are less likely to encounter traditional barriers to behavior change (e.g., motivation, self-efficacy; Rothman et al., 2011). As the current work recruited already sedentary individuals - a sample with a strengthened SB habit (i.e., $\geq 7$ hours/day) - it is likely that the reflective, motivational constructs involved in behavioural initiation process of being less sedentary (e.g., self-efficacy, outcome expectancies, intention) were not strong enough to counter the developed, automatic habit of SB. Finally, as the present study was advertised as simply a "behaviour change study", it is unlikely that the participants recruited necessarily had a pre-existing intention to change their SB. Intention is hypothesized to be the strongest predictor of behaviour during the motivational phase of the HAPA (Schwarzer, 2008); hence the effectiveness of an intervention targeting action and coping planning (i.e., post-intentional constructs) may have been limited.

There are several modifications that may improve the effectiveness of the present intervention to reduce device-measured and self-reported SB. Inclusion of components of previously successful SB interventions, such as prompts and cues, can address the lack of cognitive awareness associated with habitual behaviors, like SBs. Specific to the current intervention, the behavioural counseling was likely too brief for the action and coping plan to remain salient to participants, despite attempting to trigger an environmental cue via hanging the action plan in a high-trafficked location. The use of a brief daily prompt (e.g., via SMS) may have triggered the reflective processes needed to reduce SB. Similarly, 


\begin{tabular}{|c|c|c|c|c|c|c|}
\hline \multirow[b]{2}{*}{ TIME } & \multicolumn{3}{|c|}{ TREATMENT $(n=17)$} & \multicolumn{3}{|c|}{ CONTROL $(n=14)$} \\
\hline & MEAN & $S D$ & $95 \% C I$ & MEAN & $S D$ & $95 \% C I$ \\
\hline \multicolumn{7}{|c|}{ Self-Compared Weekly Sitting } \\
\hline Baseline & 3.47 & 0.62 & {$[3.15,3.79]$} & 3.29 & 0.91 & {$[2.76,3.81]$} \\
\hline Intervention & 2.82 & 1.13 & {$[2.24,3.41]$} & 3.50 & 0.76 & {$[3.06,3.94]$} \\
\hline Follow-up & 3.18 & 0.81 & {$[2.76,3.59]$} & 3.21 & 1.12 & {$[2.57,3.86]$} \\
\hline \multicolumn{7}{|c|}{ Self-Compared Weekly Break Frequency } \\
\hline Baseline & 2.94 & 0.83 & {$[2.52,3.37]$} & 2.79 & 0.70 & {$[2.38,3.19]$} \\
\hline Intervention & 3.89 & 0.34 & {$[3.72,4.07]$} & 2.86 & 1.03 & {$[2.26,3.45]$} \\
\hline Follow-up & 3.29 & 0.77 & {$[2.90,3.69]$} & 2.64 & 0.63 & {$[2.28,3.01]$} \\
\hline \multicolumn{7}{|c|}{ Self-Compared Weekly Break Duration } \\
\hline Baseline & 3.08 & 0.60 & {$[2.77,3.39]$} & 2.93 & 0.47 & {$[2.67,3.20]$} \\
\hline Intervention & 3.71 & 0.59 & {$[3.40,4.01]$} & 2.71 & 0.91 & {$[2.19,3.24]$} \\
\hline Follow-up & 3.29 & 0.59 & {$[2.99,3.60]$} & 2.93 & 0.47 & {$[2.66,3.20]$} \\
\hline \multicolumn{7}{|c|}{ Average Weekday Sitting Time (hours/day) } \\
\hline Baseline $^{\dagger \pi}$ & 8.39 & 2.97 & {$[6.81,9.97]$} & 7.42 & 1.79 & {$[6.28,8.56]$} \\
\hline Intervention $n^{\ddagger}$ & 7.83 & 2.54 & {$[6.42,9.24]$} & 8.46 & 2.44 & {$[6.91,10.01]$} \\
\hline Follow-up $¥ \pi$ & 7.25 & 2.30 & {$[5.97,8.53]$} & 8.17 & 2.68 & {$[6.46,9.87]$} \\
\hline
\end{tabular}

Table 3 Means, standard deviations, and 95\% confidence intervals for self-compared and average weekday self-reported sedentary behaviors throughout the study.

Note: Bold text indicates significant differences between groups at baseline $(p<0.05)$.

$\dagger=$ Treatment group $(n=16), \neq=$ Treatment group $(n=15), \uparrow=$ Treatment group $(n=12)$.

\begin{tabular}{lcccccc}
\hline \multicolumn{1}{c}{ TREATMENT $(n=17)$} & \multicolumn{3}{l}{ CONTROL $(n=14)$} \\
\hline TIME & MEAN & SD & $95 \%$ CI & MEAN & SD & $95 \%$ CI \\
\hline Positive Affect & & & & & & \\
\hline Baseline & 31.29 & 6.33 & {$[28.04,34.55]$} & 30.86 & 7.48 & {$[26.54,35.18]$} \\
\hline Intervention & 32.47 & 6.25 & {$[29.26,35.68]$} & 27.93 & 8.48 & {$[23.03,32.83]$} \\
\hline Follow-up & 32.06 & 7.08 & {$[28.42,35.70]$} & 30.86 & 8.151 & {$[26.15,35.56]$} \\
\hline Negative Affect & & & & & & \\
\hline Baseline & 20.76 & 8.36 & {$[16.47,25.06]$} & 23.00 & 7.17 & {$[18.86,27.14]$} \\
\hline Intervention & 19.76 & 5.99 & {$[16.69,22.84]$} & 20.79 & 6.85 & {$[16.83,24.74]$} \\
\hline Follow-up & 18.29 & 8.53 & {$[13.91,22.68]$} & 19.79 & 7.08 & {$[15.70,23.88]$} \\
\hline Life Satisfaction & & & & & & \\
\hline Baseline & 23.88 & 6.78 & {$[20.40,27.37]$} & 22.36 & 6.88 & {$[18.39,26.33]$} \\
\hline Intervention & 24.76 & 6.95 & {$[21.19,28.34]$} & 21.21 & 7.45 & {$[16.91,25.52]$} \\
\hline Follow-up & 24.59 & 7.91 & {$[20.52,28.66]$} & 21.36 & 7.21 & {$[17.20,25.52]$} \\
\hline Subjective Vitality & & & & & & \\
\hline Baseline & 26.18 & 7.70 & {$[22.22,30.14]$} & 24.14 & 5.46 & {$[20.99,27.30]$} \\
\hline Intervention & 27.24 & 6.24 & {$[24.03,30.44]$} & 22.93 & 8.67 & {$[17.92,27.93]$} \\
\hline Follow-up & 27.71 & 6.76 & {$[24.23,31.18]$} & 25.50 & 6.81 & {$[21.57,29.43]$} \\
\hline Overall Subjective Well-Being & & & & & \\
\hline Baseline & 48.47 & 8.02 & {$[44.34,52.60]$} & 48.00 & 8.49 & {$[43.10,52.90]$} \\
\hline Intervention & 50.76 & 7.60 & {$[46.86,54.67]$} & 44.29 & 13.46 & {$[36.51,52.06]$} \\
\hline Follow-up & 50.82 & 7.73 & {$[46.85,54.80]$} & 47.21 & 10.45 & {$[41.18,53.25]$} \\
\hline & & & & & & \\
\hline & & & & & & \\
\hline
\end{tabular}

Table 4 Means, standard deviations, and 95\% confidence intervals for outcomes of subjective well-being throughout the study. Note: Bold text indicates significant differences between groups at baseline $(p<0.05)$. 


\begin{tabular}{|c|c|c|c|c|c|c|c|c|}
\hline & & $F$ & HYP. DF & ERR. DF & $P$ & $\Lambda$ & $\eta_{\mathrm{p}}^{2}$ & $1-\beta$ \\
\hline \multicolumn{9}{|l|}{ Device-Measured Sedentary Behavior } \\
\hline \multirow[t]{2}{*}{ Average daily steps } & Interaction & 0.015 & 1 & 28 & 0.902 & 0.99 & 0.001 & 0.052 \\
\hline & Main effect & 0.012 & 1 & 28 & 0.913 & 1.00 & 0.000 & 0.051 \\
\hline \multirow[t]{2}{*}{ Average daily standing time } & Interaction & 2.686 & 2 & 28 & 0.086 & 0.84 & 0.161 & 0.489 \\
\hline & Main effect & 2.323 & 2 & 28 & 0.142 & 0.86 & 0.142 & 0.431 \\
\hline \multirow[t]{2}{*}{ Average daily stepping time } & Interaction & 0.250 & 2 & 28 & 0.780 & 0.99 & 0.006 & 0.085 \\
\hline & Main effect & 6.747 & 2 & 28 & 0.004 & 0.99 & 0.001 & 0.886 \\
\hline \multirow[t]{2}{*}{ Average daily sitting time } & Interaction & 0.630 & 2 & 28 & 0.540 & 0.95 & 0.043 & 0.145 \\
\hline & Main effect & 0.699 & 2 & 28 & 0.506 & 0.96 & 0.048 & 0.156 \\
\hline \multirow[t]{2}{*}{ Average daily sit-to-stand transitions per waking hour } & Interaction & 0.067 & 2 & 28 & 0.936 & 1.00 & 0.005 & 0.059 \\
\hline & Main effect & 1.166 & 2 & 28 & 0.326 & 0.92 & 0.077 & 0.235 \\
\hline \multicolumn{9}{|l|}{ Self-Compared Sedentary Behavior } \\
\hline \multirow[t]{2}{*}{ Self-compared weekly sitting } & Interaction & 2.286 & 2 & 28 & 0.120 & 0.86 & 0.140 & 0.425 \\
\hline & Main effect & 0.490 & 2 & 28 & 0.618 & 0.97 & 0.034 & 0.122 \\
\hline \multirow[t]{2}{*}{ Self-compared weekly break frequency } & Interaction & 2.780 & 2 & 28 & 0.079 & 0.83 & 0.166 & 0.503 \\
\hline & Main effect & 3.783 & 2 & 28 & 0.035 & 0.79 & 0.213 & 0.641 \\
\hline \multirow[t]{2}{*}{ Self-compared weekly break duration } & Interaction & 3.131 & 2 & 28 & 0.059 & 0.82 & 0.183 & 0.555 \\
\hline & Main effect & 1.067 & 2 & 28 & 0.358 & 0.93 & 0.071 & 0.218 \\
\hline \multicolumn{9}{|l|}{ Average Weekday Sitting Time } \\
\hline \multirow[t]{2}{*}{ Average Weekday Sitting Time } & Interaction & 1.725 & 2 & 24 & 0.199 & 0.87 & 0.126 & 0.326 \\
\hline & Main effect & 0.504 & 2 & 28 & 0.610 & 0.96 & 0.040 & 0.123 \\
\hline \multicolumn{9}{|l|}{ Outcomes of Subjective Well-Being } \\
\hline \multirow[t]{2}{*}{ Positive affect } & Interaction & 1.524 & 2 & 28 & 0.235 & 0.90 & 0.098 & 0.296 \\
\hline & Main effect & 0.679 & 2 & 28 & 0.515 & 0.95 & 0.046 & 0.153 \\
\hline \multirow[t]{2}{*}{ Negative affect } & Interaction & 0.109 & 2 & 28 & 0.897 & 0.99 & 0.008 & 0.065 \\
\hline & Main effect & 3.887 & 2 & 28 & 0.032 & 0.78 & 0.217 & 0.654 \\
\hline \multirow[t]{2}{*}{ Life satisfaction } & Interaction & 2.293 & 2 & 28 & 0.120 & 0.86 & 0.141 & 0.426 \\
\hline & Main effect & 0.049 & 2 & 28 & 0.952 & 1.00 & 0.003 & 0.057 \\
\hline \multirow[t]{2}{*}{ Subjective vitality } & Interaction & 1.205 & 2 & 28 & 0.315 & 0.92 & 0.079 & 0.241 \\
\hline & Main effect & 3.068 & 2 & 28 & 0.062 & 0.82 & 0.180 & 0.546 \\
\hline \multirow[t]{2}{*}{ Overall subjective well-being } & Interaction & 3.209 & 2 & 28 & 0.064 & 0.82 & 0.178 & 0.540 \\
\hline & Main effect & 0.927 & 2 & 28 & 0.408 & 0.94 & 0.062 & 0.194 \\
\hline
\end{tabular}

Table 5 Repeated-measures interaction effects and main effects of time for device-measured, self-compared, and average weekday sedentary behavior outcomes and outcomes of subjective well-being.

Note: Bold text indicates a significant effect $(p<0.05)$. Average daily standing, sitting, and stepping time calculated as a percentage of waking time.

$\Lambda=$ Wilks' Lambda, $\eta_{p}^{2}=$ partial eta squared, $1-\beta=$ observed power.

tailoring said prompts to the participant's action plan act as an additional cue to facilitate the intended behavior change. Previous work utilizing SMS-delivered prompts and cues has shown success in reducing SB among university students (Cotten \& Prapavessis, 2016) and office workers (Rollo \& Prapavessis, 2020).
The acute nature of the intervention may also have been a limitation, in contrast to SB-inducing studies that have demonstrated that 4 days (Duvivier et al., 2017) to 2 weeks (Endrighi et al., 2016) is sufficient time to significantly increase SB. As previously stated, the behavioural processes and motivations involved in 


\begin{tabular}{lcccccccccc}
\hline & 1 & 2 & 3 & 4 & 5 & 6 & 7 & 8 & 9 & 10 \\
\hline 1. Change in average daily steps & & .482 & .931 & -.698 & -.061 & -.056 & .109 & -.059 & .082 & .025 \\
\hline 2. Change in average daily standing time & .512 & & .599 & -.894 & .470 & .237 & -.141 & .013 & .319 & .360 \\
\hline 3. Change in average daily stepping time & .929 & .624 & & -.791 & .155 & -.005 & .049 & .012 & .145 & .127 \\
\hline 4. Change in average daily sitting time & -.720 & -.864 & -.802 & & -.332 & -.237 & .103 & .000 & -.309 & -.309 \\
\hline $\begin{array}{l}\text { 5. Change in average daily sit-to-stand } \\
\text { transitions per hour }\end{array}$ & .092 & .396 & .301 & -.344 & & .247 & -.075 & .075 & .182 & .122 \\
\hline 6. Change in positive affect & & & & & & & & & & \\
\hline 7. Change in negative affect & .028 & .292 & .023 & -.281 & .106 & & -.346 & .390 & .557 & .561 \\
\hline 8. Change in life satisfaction & .237 & -.108 & .197 & .004 & .127 & -.455 & & -.315 & -.330 & -.384 \\
\hline 9. Change in subjective vitality & -.097 & .031 & -.022 & -.065 & .086 &. $\mathbf{4 0 6}$ & -.483 & &. $\mathbf{6 1 3}$ & .701 \\
\hline 10. Change in overall subjective well-being & .000 & .328 & .111 & -.310 & .153 & .597 & -.549 & .701 & .790 & \\
\hline
\end{tabular}

Table 6 Pearson correlation matrix between residuals and absolute differences of device-measured SB outcomes and outcomes of SWB. $(n=31)$.

Note: Correlations between residuals are presented below the line; correlations between absolute differences are presented above the line. Average daily standing, sitting, and stepping time calculated as a percentage of waking time.

Bold text indicates a significant correlation ( $p<0.05)$; bold, italicized text indicates a significant correlation $(p<0.01)$.

\begin{tabular}{lccccccccc}
\hline & 1 & 2 & 3 & $4^{\ddagger}$ & 5 & 6 & 7 & 8 & 9 \\
\hline 1. Self-compared weekly sitting & & -.440 & -.473 & .393 & -.286 & .017 & -.298 & -.238 & -.319 \\
\hline 2. Self-compared weekly break frequency & -.251 & & .600 & -.428 & .277 & -.089 & .323 & .144 & .206 \\
\hline 3. Self-compared weekly break duration & -.326 & .768 & & -.349 & .450 & -.125 & .486 & .402 & .392 \\
\hline 4. Average weekday sitting & .317 & -.263 & -.061 & & -.236 & -.008 & -.531 & -.376 & -.320 \\
\hline 5. Positive affect & -.036 & .307 & .353 & -.203 & & -.346 & .390 & .557 & .561 \\
\hline 6. Negative affect & -.050 & -.298 & -.194 & .110 & -.455 & & -.315 & -.330 & -.384 \\
\hline 7. Life satisfaction & -.297 & .360 & .419 & -.475 & .406 & -.483 & & .613 & .701 \\
\hline 8. Subjective vitality & -.164 & .279 & .331 & -.312 & .586 & -.494 & .629 & & .792 \\
\hline 9. Overall subjective well-being & -.330 & .360 & .328 & -.377 & .597 & -.549 & .701 & .790 & \\
\hline
\end{tabular}

Table 7 Pearson correlation matrix between residuals and absolute differences of self-compared and average weekday SB and outcomes of SWB. $(n=31)$.

Note: Correlations between residuals are presented below the line; correlations between absolute differences are presented above the line.

Bold text indicates a significant correlation $(p<0.05)$; bold, italicized text indicates a significant correlation $(p<0.01)$.

$\ddagger=(n=27)$.

performing an automatic behaviour, like SB, likely differ from those reflective and cognitive processes associated with reducing SB (i.e., alternative behaviour). Previous work utilizing this intervention in a university student population did not observe differences in SB outcomes between groups until after the third week of intervention, following the follow-up behavioral session (Sui \& Prapavessis, 2018). Thus, longer intervention periods may be necessary to observe changes in habitual changes in SB. In addition, follow-up behavioral sessions (e.g., the week proceeding the first behavioral session) can promote the use of feedback, review of previous behavior, intention formation as behavioral strategies. Together, increased contact and follow-up may improve the effectiveness of the current intervention through facilitating behavioural initiation (Rothman et al., 2011). Finally, measures used to assess SB may not be sensitive enough to capture changes in SWB. Previous work suggests that daily changes in SB impact SB outcomes (Maher et al., 2014; Maher \& Conroy, 2017). Hence, adapting present instruments into previous-day recall questionnaires can provide a more accurate depiction of SB (Matthew et al., 2013) and help to capture relationships between daily changes in SB and outcomes of SWB. 


\section{INTERVENTION EFFECTIVENESS - IMPROVING SUBJECTIVE WELL-BEING}

Four out of five SWB outcomes (i.e., positive affect, life satisfaction, subjective vitality, overall SWB) demonstrated small-to-medium-sized interaction effects (i.e., $0.079 \leq \eta_{p}{ }^{2} \leq 0.178$ ) favoring the treatment group, which indicates promise for the intervention in improving these SWB outcomes; however, as a whole, the intervention was ineffective in modifying this outcome. The lack of change observed among positive and negative affect may be explained, in part, by their measurement. Specifically, changes in state affect elicited by changes in SB may not be captured through weekly recall; rather, daily recall measurements may be necessary to capture these relationships (Maher et al., 2014; Maher \& Conroy, 2017). Conversely, changes in more global measures of SWB, such as life satisfaction and overall SWB, may be appropriately captured through weekly recall. Overall, the ineffectiveness of the current intervention to modify SB limits the interpretation of SB and SWB relationships. However, consistent moderate strength correlations between self-reported SBs and SWB outcomes support the previous work of Maher and colleagues (Maher et al., 2014; Maher \& Conroy, 2017) and Okely et al., (2019), in that changes in perceived SB may be more influential for improving SWB outcomes than objective changes in $S B$. Future intervention research should explore which SB outcomes elicit the greatest improvements in SWB.

\section{LIMITATIONS}

Although sample size calculations are not typically part of pilot studies, the low observed power statistics and trending $p$ values indicate that the current work was underpowered. Drawing on previous studies that were successful in reducing SB using a similar HAPA-based intervention (Rollo \& Prapavessis, 2020; Sui \& Prapavessis, 2018), future iterations of the present work should aim for a sample size of 50-60 participants (i.e., 25-30 participants per group) through longer recruitment periods or recruitment over multiple semesters, in addition to the inclusion of the previously outlined strategies. Blinding of participants may also have presented as a limitation. Efforts were made to blind group allocation from participants (i.e., advertising the study as a "behaviour change study") and anecdotally, the majority of participants mentioned they were unaware of the purpose of the study. However, some participants may have perceived the purpose of the study, which in turn may have influenced their devicemeasured SB and self-reported outcomes. Further, the lack of allocation blinding to the researcher presents as another limitation. Although efforts were made by the researcher to allocate randomly, the lack of concealment may have affected the delivery of the study. Utilizing third-party sequence blinding and sealed envelopes can ensure sequence and allocation concealment.

\section{GENERALIZABILITY}

Given the pilot nature of the present trial, the generalizability of these findings is limited. This trial needs replication with larger and more heterogeneous samples before definitive conclusions are drawn.

\section{INTERPRETATION}

Overall, the present randomized pilot trial provides evidence that the current acute behavioral intervention was ineffective in reducing device-measured and self-reported SB in a sample of university students over a 1-week period. Compared to previous work, this ineffectiveness may be explained by behavioural differences in reducing vs. inducing SB, strong automatic processes for sustaining SB, and/or a weak intention to reduce SB among participants. This ineffectiveness may also be in part due to the pilot study being underpowered to show statistical significance for some of the variables of interest. Together, with the addition of longer intervention periods, increased contact through followup sessions, and prompts/cues, the current intervention may relay greater effectiveness in reducing SB in future trials.

\section{REGISTRATION AND PROTOCOL}

The following trial and associated protocol are registered at ClinicalTrials.gov under ClinicalTrials.gov ID: NCT03694951 and Protocol ID: 112399, respectively.

\section{ADDITIONAL FILES}

The additional files for this article can be found as follows:

- Supplementary Material 1. CONSORT 2010

checklist of information to include when reporting a randomised trial*. DOI: https://doi.org/10.5334/hpb.29.s1

- Supplementary Material 2. Modified SIT-Q 7d Questionnaire. DOI: https://doi.org/10.5334/hpb.29.s2

- Supplementary Material 3. Means, standard deviations, and $95 \%$ confidence intervals for selfcompared, average weekday, and domain-specific self-reported sedentary behaviors throughout the study. DOI: https://doi.org/10.5334/hpb.29.s3

- Supplementary Material 4. Repeated-measures interaction effects and main effects of time for domain-specific sedentary behavior outcomes. DOI: https://doi.org/10.5334/hpb.29.s4

- Supplementary Material 5. Pearson correlations between residuals and absolute differences of domain-specific SBs and outcomes of SWB. $(n=31)$. DOI: https://doi.org/10.5334/hpb.29.s5 


\section{FUNDING INFORMATIONS}

The following trial was funded by a Social Sciences and Humanities Research Council of Canada Insight Development Grant under award number: 430-2018-00886.

\section{COMPETING INTERESTS}

The authors denote no other perceived or actual conflicts of interest with the trial.

\section{AUTHOR AFFILIATIONS}

Wuyou Sui (iD) orcid.org/0000-0002-2171-7989

Western University, CA

Harry Prapavessis (D) orcid.org/0000-0003-0636-7316

Western University, CA

\section{REFERENCES}

Aliabad, H. O., Vafaeinasab, M., Morowatisharifabad, M. A., Afshani, S. A., Firoozabadi, M. G., \& Forouzannia, S. K. (2014). Maintenance of Physical Activity and Exercise Capacity After Rehabilitation in Coronary Heart Disease: A Randomized Controlled Trial. Global Journal of Health Science, 6(6). DOI: https://doi.org/10.5539/gjhs.v6n6p198

Bakeman, R. (2005). Recommended effect size statistics for repeated measures designs. Behavior Research Methods, 37(3), 379-384. DOI: https://doi.org/10.3758/BF03192707

Bostic, T. J., McGartland Rubio, D., \& Hood, M. (2000). A Validation of the Subjective Vitality Scale Using Structural Equation Modeling. Social Indicators Research, 52(3), 313324. DOI: https://doi.org/10.1023/A:1007136110218

Burnet, K., Higgins, S., Kelsch, E., Moore, J. B., \& Stoner, L. (2020). The effects of manipulation of Frequency, Intensity, Time, and Type (FITT) on exercise adherence: A meta analysis. Translational Sports Medicine, 3(3), 222-234. DOI: https://doi.org/10.1002/tsm2.138

Castro, O., Bennie, J., Vergeer, I., Bosselut, G., \& Biddle, S. J. H. (2020). How Sedentary Are University Students? A Systematic Review and Meta-Analysis. Prevention Science, 21(3), 332-343. DOI: https://doi.org/10.1007/s11121-02001093-8

Colley, R. C., Garriguet, D., Janssen, I., Craig, C. L., Clarke, J., \& Tremblay, M. S. (2011). Physical activity of Canadian adults: Accelerometer results from the 2007 to 2009 Canadian Health Measures Survey. Statistics Canada, 22(1). DOI: https://doi.org/10.1016/j.yspm.2011.03.006

Cotten, E., \& Prapavessis, H. (2016). Increasing Nonsedentary Behaviors in University Students Using Text Messages: Randomized Controlled Trial. JMIR MHealth and UHealth, 4(99), 1-11. DOI: https://doi.org/10.2196/mhealth.5411

Craig, C. L., Marshall, A. L., Sjöström, M., Bauman, A. E., Booth, M. L., Ainsworth, B. E., Pratt, M., Ekelund, U.,
Yngve, A., Sallis, J. F., \& Oja, P. (2003). International physical activity questionnaire: 12 -Country reliability and validity. Medicine and Science in Sports and Exercise, 35(8), 1381-1395. DOI: https://doi.org/10.1249/01. MSS.0000078924.61453.FB

De Rezende, L. F. M., Lopes, M. R., Rey-Loṕez, J. P., Matsudo, V. K. R., \& Luiz, O. D. C. (2014). Sedentary behavior and health outcomes: An overview of systematic reviews. PLOS ONE, 9(8). DOI: https://doi.org/10.1371/journal.pone.0105620

Diener, E., Emmons, R. A., Larsen, R. J., \& Griffin, S. (1985). The Satisfaction With Life Scale. Journal of Personality Assessment, 49(1), 71-75. DOI: https://doi.org/10.1207/ s15327752jpa4901_13

Diener, E., Suh, E. M., Lucas, R. E., \& Smith, H. L. (1999). Subjective well-being: Three decades of progress. Psychological Bulletin, 125(2), 276-302. https://doi. org/10.1037/0033-2909.125.2.276

Duan, B. (1998). The robustness of trimming and Winsorization when the population distribution is skewed. In Dissertation Abstracts International, Volume: 59-09, Section: B,. Tulane University.

Duan, Y. P., Wienert, J., Hu, C., Si, G. Y., \& Lippke, S. (2017). Web-Based Intervention for Physical Activity and Fruit and Vegetable Intake Among Chinese University Students: A Randomized Controlled Trial. Journal of Medical Internet Research, 19(4), e106. DOI: https://doi.org/10.2196/jmir.7152

Duvivier, B. M. F. M., Schaper, N. C., Koster, A., van Kan, L., Peters, H. P. F., Adam, J. J., Giesbrecht, T., Kornips, E., Hulsbosch, M., Willems, P., Hesselink, M. K. C., Schrauwen, P., \& Savelberg, H. H. C. M. (2017). Benefits of Substitution Sitting with Standing and Walking in Free-living Conditions for Cardiometabolic Risk Markers, cognition and Mood in Overweight Adults. Frontiers in Psychology, 8. DOI: https:// doi.org/10.3389/fphys.2017.00353

Edwards, M. K., \& Loprinzi, P. D. (2016). Effects of a Sedentary Behavior-Inducing Randomized Controlled Intervention on Depression and Mood Profile in Active Young Adults. Mayo Clinic Proceedings, 91(8), 984-998. DOI: https://doi. org/10.1016/j.mayocp.2016.03.021

Edwards, M. K., \& Loprinzi, P. D. (2017). Experimentally increasing sedentary behavior results in decreased life satisfaction. Health Promotion Perspectives, 7(2), 88-94. DOI: https://doi.org/10.15171/hpp.2017.16

Edwardson, C. L., Winkler, E. A. H., Bodicoat, D. H., Yates, T., Davies, M. J., Dunstan, D. W., \& Healy, G. N. (2017). Considerations when using the activPAL monitor in field-based research with adult populations. Journal of Sport and Health Science, 6(2), 162-178. DOI: https://doi. org/10.1016/j.jshs.2016.02.002

Endrighi, R., Steptoe, A., \& Hamer, M. (2016). The effect of experimentally induced sedentariness on mood and psychobiological responses to mental stress. The British Journal of Psychiatry: The Journal of Mental Science, 208(3), 245-251. DOI: https://doi.org/10.1192/bjp.bp.114.150755

Hogan, C. L., Catalino, L. I., Mata, J., \& Fredrickson, B. L. (2015). Beyond emotional benefits: Physical activity and 
sedentary behaviour affect psychosocial resources through emotions. Psychology \& Health, 30(3), 354-369. DOI: https://doi.org/10.1080/08870446.2014.973410

Huta, V. (2015). An overview of hedonic and eudaimonic wellbeing concepts.

Koster, A., Shiroma, E. J., Caserotti, P., Matthews, C. E., Chen, K. Y., Glynn, N. W., \& Harris, T. B. (2016). Comparison of Sedentary Estimates between activPAL and Hip- and Wrist-Worn ActiGraph. Medicine \& Science in Sports \& Exercise, 48(8), 1514-1522. DOI: https://doi.org/10.1249/ MSS.0000000000000924

Ku, P.-W., Steptoe, A., Liao, Y., Hsueh, M.-C., \& Chen, L.-J. (2018). A cut-off of daily sedentary time and all-cause mortality in adults: a meta-regression analysis involving more than 1 million participants. BMC Medicine, 16(1), 74. DOI: https://doi.org/10.1186/s12916-018-1062-2

Lippke, S., Ziegelmann, J. P., \& Schwarzer, R. (2005). Stage-specific adoption and maintenance of physical activity: Testing a three-stage model. Psychology of Sport and Exercise. DOI: https://doi.org/10.1016/j. psychsport.2004.11.002

Maher, J. P., \& Conroy, D. E. (2017). Daily Life Satisfaction in Older Adults as a Function of (In)Activity. The Journals of Gerontology: Series B, 72(4), 593-602. DOI: https://doi. org/10.1093/geronb/gbv086

Maher, J. P., Doerksen, S. E., Elavsky, S., \& Conroy, D. E. (2014) Daily satisfaction with life is regulated by both physical activity and sedentary behavior. Journal of Sport \& Exercise Psychology, 36(2), 166-178. DOI: https://doi.org/10.1123/ jsep.2013-0185

Matthew, C. E., Kozey-Keadle, S., Sampson, J., Lyden, K., Bowles, H. R., Moore, S. C., Libertine, A., Freedson, P. S., \& Fowke, J. H. (2013). Validation of a Previous-Day Recall Measure of Active and Sedentary Behaviors. Medicine \& Science in Sports \& Exercise, 45(8), 1629-1638. DOI: https:// doi.org/10.1249/MSS.0b013e3182897690

Michie, S., Richardson, M., Johnston, M., Abraham, C., Francis, J., Hardeman, W., Eccles, M. P., Cane, J., \& Wood, C. E. (2013). The Behavior Change Technique Taxonomy (v1) of 93 Hierarchically Clustered Techniques: Building an International Consensus for the Reporting of Behavior Change Interventions. Annals of Behavioral Medicine, 46(1), 81-95. DOI: https://doi.org/10.1007/s12160-013-9486-6

Mittelmark, M. B., \& Bauer, G. F. (2017). The Meanings of Salutogenesis. In The Handbook of Salutogenesis (pp. 7-13). Springer International Publishing. DOI: https://doi. org/10.1007/978-3-319-04600-6_2

Moher, D., Hopewell, S., Schulz, K. F., Montori, V., Gøtzsche, P. C., Devereaux, P. J., Elbourne, D., Egger, M., \& Altman, D. G. (2012). CONSORT 2010 explanation and elaboration: Updated guidelines for reporting parallel group randomised trials. International Journal of Surgery, 10(1), 28-55. DOI: https://doi.org/10.1016/j.ijsu.2011.10.001

O'Neill, C., \& Dogra, S. (2016). Different Types of Sedentary Activities and Their Association With Perceived Health and Wellness Among Middle-Aged and Older Adults:
A Cross-Sectional Analysis. American Journal of Health Promotion, 30(5), 314-322. DOI: https://doi. org/10.1177/0890117116646334

Okely, J. A., Cukic, I., Shaw, R. J., Chastin, S. F., Dall, P. M., Deary, I. J., Der, G., Dontje, M. L., Skelton, D. A., Gale, C. R., Čukić, I., Shaw, R. J., Chastin, S. F., Dall, P. M., Deary, I. J., Der, G., Dontje, M. L., Skelton, D. A., \& Gale, C. R. (2019). Positive and negative well-being and objectively measured sedentary behaviour in older adults: evidence from three cohorts. BMC Geriatrics, 19(1), 1-10. DOI: https://doi. org/10.1186/s12877-019-1026-1

PAL Technologies. (2020). PAL family. http://www.palt.com/ pals/.

RANDOM.org. (2020). List Randomizer. https://www.random. org/lists/.

Rollo, S., \& Prapavessis, H. (2020). A Combined Health Action Process Approach and mHealth Intervention to Increase NonSedentary Behaviours in OfficeWorking Adults-A Randomised Controlled Trial. Applied Psychology: Health and Well-Being, aphw.12201. DOI: https://doi.org/10.1111/ aphw.12201

Rothman, A. J., Baldwin, A. S., Hertel, A. W., \& Fuglestad, P. T. (2011). Self-regulation and behavior change: Disentangling behavioral initiation and behavioral maintenance.

Ryan, R. M., \& Deci, E. L. (2001). On Happiness and Human Potentials: A Review of Research on Hedonic and Eudaimonic Well-Being. Annual Review of Psychology, 52(1), 141-166. DOI: https://doi.org/10.1146/annurev. psych.52.1.141

Ryan, R. M., \& Frederick, C. (1997). On Energy, Personality, and Health: Subjective Vitality as a Dynamic Reflection of Well-Being. Joumal of Personality, 65, 28-31. September 1997. DOI: https://doi.org/10.1111/j.1467-6494.1997. tb00326.x

Schwarzer, R. (2008). Modeling health behavior change: How to predict and modify the adoption and maintenance of health behaviors. Applied Psychology, 57(1), 1-29. DOI: https://doi.org/10.1111/j.1464-0597.2007.00325.x

Sui, W., \& Prapavessis, H. (2016). Testing the face validity and reliability of a modified SIT-Q 7 day recall questionnaire measuring sedentary time and break frequency $\&$ duration. Journal of Sport and Exercise Psychology, 38, S262.

Sui, W., \& Prapavessis, H. (2018). Standing Up for Student Health: An Application of the Health Action Process Approach for Reducing Student Sedentary Behavior-Randomised Control Pilot Trial. Applied Psychology. Health and Well-Being, 10(1), 87-107. DOI: https://doi.org/10.1111/aphw.12105

Tennant, R., Hiller, L., Fishwick, R., Platt, S., Joseph, S., Weich, S., Parkinson, J., Secker, J., \& Stewart-Brown, S. (2007). The Warwick-Edinburgh mental well-being scale (WEMWBS): Development and UK validation. Health and Quality of Life Outcomes, 5, 1-13. DOI: https://doi. org/10.1186/1477-7525-5-63

Tremblay, M. S., Aubert, S., Barnes, J. D., Saunders, T. J., Carson, V., Latimer-Cheung, A. E., Chastin, S. F. M., Altenburg, T. M., \& Chinapaw, M. J. M. (2017). Sedentary 
Behavior Research Network (SBRN) - Terminology Consensus Project process and outcome. The International Journal of Behavioral Nutrition and Physical Activity, 14, 17. DOI: https://doi.org/10.1186/s12966-017-0525-8

Watson, D., Clark, L. A., \& Tellegen, A. (1988). Development and Validation of Brief Measures of Positive and Negative Affect: The PANAS Scales. Journal of Personality and Social Psychology, 54(6), 1063-1070. DOI: https://doi. org/10.1037/0022-3514.54.6.1063

Wijndaele, K., De Bourdeaudhuij, I., Godino, J. G., Lynch, B. M., Griffin, S. J., Westgate, K., \& Brage, S. (2014). Reliability and validity of a domain-specific last 7-d sedentary time questionnaire. Medicine and Science in Sports and Exercise. DOI: https://doi.org/10.1249/MSS.0000000000000214
Wrosch, C., \& Sabiston, C. M. (2013). Goal adjustment, physical and sedentary activity, and well-being and health among breast cancer survivors. Psycho-Oncology, 22(3), 581-589. DOI: https://doi.org/10.1002/pon.3037

\section{PEER REVIEW COMMENTS}

Health Psychology Bulletin has blind peer review, which is unblinded upon article acceptance. The editorial history of this article can be downloaded here:

- PR File 1. Peer Review History. DOI: https://doi. org/10.5334/hpb.29.pr1

\section{TO CITE THIS ARTICLE:}

Sui, W. \& Prapavessis, H. (2021). The Effect of an Acute Sedentary Behaviour Reducing Intervention on Subjective Well-Being among University Students: A Pilot Randomized Trial. Health Psychology Bulletin, 5(1), pp. 46-60. DOI: https://doi.org/10.5334/hpb.29

Submitted: 10 December 2020 Accepted: 07 February 2021 Published: 12 March 2021

\section{COPYRIGHT:}

(c) 2021 The Author(s). This is an open-access article distributed under the terms of the Creative Commons Attribution 4.0 International License (CC-BY 4.0), which permits unrestricted use, distribution, and reproduction in any medium, provided the original author and source are credited. See http://creativecommons.org/licenses/by/4.0/.

Health Psychology Bulletin is a peer-reviewed open access journal published by Ubiquity Press. 